\title{
A randomized, double-blind, placebo-controlled Phase III trial of duloxetine in Japanese patients with knee pain due to osteoarthritis
}

This article was published in the following Dove Press journal: Journal of Pain Research

\author{
Yuji Uchio' \\ Hiroyuki Enomoto ${ }^{2}$ \\ Levent Alev² \\ Yuki Kato ${ }^{3}$ \\ Hiroyuki Ishihara ${ }^{3}$ \\ Toshinaga Tsuji ${ }^{4}$ \\ Toshimitsu Ochiai ${ }^{5}$ \\ Shinichi Konno ${ }^{6}$ \\ 'Department of Orthopaedic \\ Surgery, Shimane University School \\ of Medicine, Shimane, Japan; ${ }^{2} \mathrm{Bio}-$ \\ Medicine, Medicines Development \\ Unit, Eli Lilly Japan K.K., Kobe, Japan \\ ${ }^{3}$ Clinical Development Department, \\ Shionogi \& Co., Ltd., Osaka, Japan; \\ ${ }^{4}$ Medical Affairs Department, \\ Shionogi \& Co., Ltd., Osaka, Japan; \\ ${ }^{5}$ Biostatistics Department, Shionogi \& \\ Co., Ltd., Osaka, Japan; ${ }^{6}$ Department \\ of Orthopaedic Surgery, Fukushima \\ Medical University School of \\ Medicine, Fukushima, Japan
}

Correspondence: Yuji Uchio

Department of Orthopaedic Surgery, Shimane University School of Medicine, 89-I Enya-cho, Izumo, Shimane 693-850I, Japan

Tel +8I 853202242

Fax +8I 853202236

Email orthop@med.shimane-u.ac.jp
Purpose: To examine the efficacy and safety of duloxetine in Japanese patients with knee pain due to osteoarthritis.

Patients and methods: Patients were randomized to receive duloxetine $60 \mathrm{mg} /$ day or placebo for 14 weeks in a double-blind manner (ClinicalTrials.gov Identifier: NCT02248480). The primary efficacy endpoint was mean change in Brief Pain Inventory pain severity (BPI-Severity) average pain. Secondary endpoints included improvement in other BPI-Severity scales, Patient Global Impression of Improvement, Clinical Global Impressions of Severity, health-related quality of life (HRQoL) scales, range of motion of the knee joint, safety and tolerability, and structural changes on X-ray images.

Results: Of the 354 randomized patients, 161 in the duloxetine group and 162 in the placebo group completed the study. BPI-Severity average pain improved significantly with duloxetine vs. placebo ( -2.57 vs. -1.80 ; adjusted mean difference: $-0.77 ; 95 \% \mathrm{CI}:-1.11$ to $-0.43 ; P<0.0001)$. Secondary efficacy endpoints and most HRQoL scales showed greater improvements in the duloxetine group than the placebo group. Adverse events observed in $\geq 5 \%$ of patients that were more frequent in the duloxetine than placebo group were somnolence, constipation, dry mouth, nausea, malaise, and decreased appetite. There were no marked changes in range of motion of the knee joint (efficacy), X-ray images, or Kellgren-Lawrence grade (safety) in either group. Conclusion: Duloxetine reduced pain and improved function in patients with knee osteoarthritis, without causing X-ray abnormalities or altered knee joint mobility. Reduced pain was associated with improved HRQoL. Adverse events were consistent with duloxetine's known safety profile. Keywords: double-blind, randomized, placebo-controlled trial, duloxetine, osteoarthritis

\section{Introduction}

Osteoarthritis (OA) is a common, chronic degenerative disease associated with cartilage degeneration and joint deformity that leads to pain and subsequent impairments in health-related quality of life (HRQoL). Knee OA, a very common form of OA, is a leading cause of disability worldwide. ${ }^{1}$ Symptomatic knee OA from population-based studies in the USA has been reported in $12.1 \%-16.3 \%$ of older adults, which is similar to rates in Europe ${ }^{2}$ and Japan. ${ }^{3}$ However, the prevalence of knee OA increases with age and is higher in women, people with obesity, and in non-urban populations. ${ }^{2,3}$

Pain is a strong predictor of disability associated with knee $\mathrm{OA}^{4,5}$ and reduced HRQoL. ${ }^{6}$ In addition, pain experienced with OA can be further exacerbated by pain avoidance strategies that result in maladaptive movement and patients' psychosocial 
responses to pain and the disability that ensues. ${ }^{7}$ Thus, improving physical function and reducing pain in patients with knee OA are important treatment goals. ${ }^{8}$

Duloxetine is a serotonin and norepinephrine reuptake inhibitor with antidepressant, central pain inhibitory, and anxiolytic activities that is approved in the USA and other countries for chronic musculoskeletal pain, including pain associated with knee OA. In addition, the Osteoarthritis Research Society International guidelines recommend duloxetine for treatment of multiple-joint OA with or without comorbidities, and for knee-only OA in patients without comorbidities. ${ }^{8}$ Several studies conducted in the US/Europe and China have shown significant improvements in pain for patients with knee OA who were treated with duloxetine compared with placebo. ${ }^{9-11}$ However, these studies focused on pain, knee function, and HRQoL, without evaluating $\mathrm{X}$-ray images, knee range of motion (ROM), or whether the analgesic effect of duloxetine could aggravate or prolong the pathological condition of OA. Further, no studies have been conducted in Japan, where the number of people with knee OA is growing with the aging society.

This Phase III clinical study was conducted to evaluate the efficacy and safety of duloxetine in Japanese patients with knee OA. The primary objective was to determine whether duloxetine was superior to placebo in the Japanese population.

\section{Materials and methods}

\section{Study design}

This multicenter, randomized, placebo-controlled, doubleblind study was conducted at 47 medical institutions in Japan from October 2014 to June 2015 (ClinicalTrials.gov: NCT02248480).
The protocol and informed consent were approved by the Institutional Review Board of each medical institution (Box S1). The study was conducted in compliance with Good Clinical Practice, the ethical standards of the responsible committee on human experimentation (Institutional and National) and the Helsinki Declaration of 1975 (2000 revision). Written informed consent was obtained from all patients before participation.

The study phases (17-18 weeks) comprised screening (1-2 weeks), treatment (14 weeks), tapering (1 week), and follow-up (1 week; Figure 1). Tapering was conducted to avoid symptoms resulting from abrupt discontinuation of duloxetine. ${ }^{12}$ After screening, eligible patients were randomized 1:1 to receive duloxetine $\left(\right.$ Cymbalta $^{\circledR}$; Eli Lilly and Co., Indianapolis, IN, USA) or placebo with an Interactive Web Response System and stochastic minimization method using the baseline Brief Pain Inventory (BPI) pain severity (BPI-Severity) average pain $(<6, \geq 6)$ as an allocation factor.

Allowed and prohibited treatments/interventions are described in Supplementary Materials. Analgesics (including non-steroidal anti-inflammatory drugs [NSAIDs]) and other therapeutic agents for knee OA were washed out during the screening phase; their concomitant use was prohibited during the study. Drugs with analgesic effect (e.g., NSAIDs) were permitted as rescue medication for up to 3 consecutive days and a cumulative total of 20 days.

Investigators enrolled patients and dispensed the study drugs, which were identical in appearance, odor, and taste. Patients received duloxetine (20 mg capsule for 1 week, two $20 \mathrm{mg}$ capsules for 1 week, three $20 \mathrm{mg}$ capsules for 12 weeks) or placebo (matching placebo capsules for 14 weeks) once daily after breakfast. Following completion of treatment or discontinuation after 2 weeks of treatment,

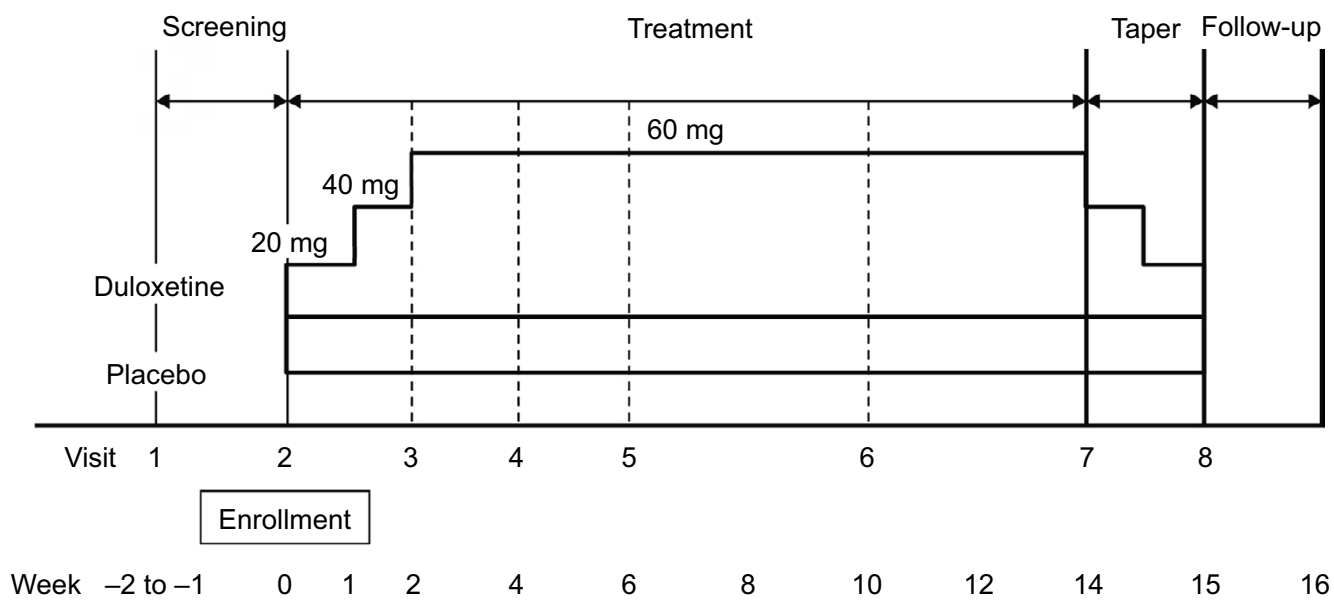

Figure I Study design. 
patients underwent tapering and then entered either the longterm extension study or the 1-week follow-up phase.

There were no changes to the study design or statistical analyses after starting enrollment and unblinding. A post hoc analysis was performed to assess the potential effect of age on BPI-Severity average pain among patients aged $<65$ and $\geq 65$ years.

\section{Patients}

Male and female outpatients aged $40-<80$ years were eligible if they had experienced pain for $\geq 14$ days/month during the 3-month period before Visit 1 and had a BPI-Severity average pain score of $\geq 4$. Patients needed to satisfy the American College of Rheumatology criteria ${ }^{13}$ for idiopathic knee OA (knee pain, bone spurs detected on plain X-ray images, and at least one of age $>50$ years, morning stiffness resolving within 30 minutes, or crepitus).

Key exclusion criteria were as follows: previous administration of duloxetine; inflammatory arthritis (e.g., rheumatoid arthritis) or autoimmune diseases (except Hashimoto's disease and type 1 diabetes); invasive treatment (e.g., joint lavage or intra-articular hyaluronic acid or steroid injections) in either knee within 1 month before Visit 1; arthroscopic surgery of the affected joint within 1 year before Visit 1 or a history of joint replacement or osteotomy; end-stage OA (e.g., patients with loss of joint space or loss of articular cartilage) or patients scheduled to undergo surgery of the affected joint during the study; major depressive disorders based on the Mini International Neuropsychiatric Interview; ${ }^{14}$ and suicidal tendencies according to the Columbia-Suicide Severity Rating Scale (C-SSRS) ${ }^{15}$

\section{Study objectives and evaluation methods}

The primary objective was to assess the efficacy of duloxetine compared with placebo in the reduction in BPI-Severity average pain. At each visit, patients evaluated their pain for each item during the past 24 hours on an 11-point rating scale (0 ["no pain"] to 10 ["pain as bad as you can imagine"]). ${ }^{16}$ The BPI is validated in patients with chronic non-malignant pain ${ }^{17}$ and has been used previously with duloxetine. ${ }^{18}$

Secondary efficacy endpoints included BPI-Severity $(30 \%, 50 \%$, and sustained reductions in average pain, worst pain, least pain, and pain right now); 24-hour BPI-Severity weekly average pain from patient diaries $(30 \%, 50 \%$, and sustained reductions, and 24-hour BPI-Severity worst pain); BPI-Interference (general activity, mood, walking ability, normal work, relations with others, sleep, and life enjoyment); Clinical Global Impressions of Severity (CGI-S); ${ }^{19}$
Patient's Global Impressions of Improvement (PGI-I); ${ }^{19}$ the European Quality of Life Questionnaire-5 Dimension (EQ5D) $;{ }^{20}$ the 36-Item Short Form Health Survey (SF-36); ${ }^{21}$ and HRQoL specific to OA assessed using the Western Ontario and McMaster Universities Osteoarthritis Index (WOMAC). ${ }^{22}$

The Outcome Measures in Rheumatology Clinical TrialsOsteoarthritis Research Society International (OMERACTOARSI) response rate ${ }^{23}$ was calculated using the 24-hour BPI-Severity average pain score, the WOMAC subscale score for "difficulty in performing daily activities," and the Patient Global Assessment of Illness.

Safety was evaluated based on treatment discontinuation rate, adverse events (AEs), serious AEs, adverse drug reactions (ADRs), clinical laboratory tests, body weight, blood pressure, pulse rate, electrocardiography, C-SSRS, and Fall Questionnaire (fall scores) from Visit 1 to the end of followup. Investigators assessed the severity of AEs/ADRs and the possibility of a causal relationship to the study drug.

Exploratory outcomes were knee ROM (measured by goniometer) as an efficacy outcome and Kellgren-Lawrence (K-L) Grading Scale for knee OA severity (evaluated in one knee by examination of radiographs) as a safety outcome (Supplementary Materials).

\section{Statistical methods}

The sample size calculation is presented in the Supplementary Materials.

The primary efficacy analysis (change in BPI-Severity average pain from baseline to Week 14 of treatment) was performed using the full analysis set, which included all randomized patients who received at least one dose of the study drug and who had BPI-Severity average pain recorded at baseline and at least once during treatment. The safety analysis set included all patients who received at least one dose of study drug.

A mixed-effects model repeated measures approach was used for the primary analysis of the primary endpoint. A linear model with an unstructured covariance structure of the error variance was applied, using the change in BPISeverity average pain from baseline as a response variable. Treatment group, time point, and treatment group-by-time point interaction were modeled as fixed effects, and baseline BPI-Severity average pain was the covariate. The degree of freedom was adjusted by a Kenward-Roger approximation. Methods used for all other efficacy and safety endpoints are presented in the Supplementary Materials.

Changes from baseline within groups are presented as the adjusted mean and $95 \% \mathrm{CI}$, and between groups as the 
adjusted mean difference (duloxetine minus placebo) with 95\% CI. All statistical tests were performed at a two-sided significance level of 0.05 , unless otherwise noted. All analyses were conducted using SAS software (version 9.2; SAS Institute, Cary, NC, USA).

\section{Results}

\section{Patient disposition and characteristics}

Of the 395 patients screened, 354 were randomized to duloxetine (178 randomized, 177 analyzed for efficacy) or placebo (176 randomized and analyzed), and 336 patients entered the taper phase. One patient in the duloxetine group did not undergo clinical assessments after starting the study drug. During treatment, 17 patients discontinued duloxetine and 14 discontinued placebo; one patient (placebo) discontinued from the taper phase (Figure 2).

Baseline characteristics were balanced between groups (Table 1), except for prior drug therapy, which was slightly more frequent in the placebo group.

\section{Efficacy}

The adjusted mean change $(95 \% \mathrm{CI})$ in BPI-Severity average pain at Week 14 was $-2.57(-2.81$ to -2.33$)$ for duloxetine and $-1.80(-2.05$ to -1.56$)$ for placebo (adjusted mean difference [95\% CI]: $-0.77[-1.11$ to -0.43$]$; $P<0.0001)$, demonstrating superiority of duloxetine over placebo (Table 2 , Figure 3). These findings were consistent for all missing data imputation methods (data not shown) and between groups at all other time points (Figure 3). The response to duloxetine was similar in men (adjusted mean difference from placebo [95\% CI]: -0.79 [ -1.50 to -0.09$] ; P=0.0276)$ and women $(-0.75[-1.14$ to -0.36$] ; P=0.0002)$.

Reductions in all secondary measures of BPI-Severity, including the proportions of patients achieving 30\%, 50\%,

Table I Baseline characteristics (full analysis set)

\begin{tabular}{|c|c|c|c|}
\hline Characteristic & $\begin{array}{l}\text { Placebo } \\
(n=176)\end{array}$ & $\begin{array}{l}\text { Duloxetine } \\
(n=177)\end{array}$ & $P_{\text {-value }}{ }^{a}$ \\
\hline Age, years & $66.4 \pm 8.4$ & $65.5 \pm 8.0$ & $0.3133^{b}$ \\
\hline Male & $44(25.0 \%)$ & 35 (19.8\%) & $0.2526^{c}$ \\
\hline Female & $132(75.0 \%)$ & $142(80.2 \%)$ & \\
\hline Weight, kg & $62.56 \pm 11.53$ & $62.71 \pm 12.85$ & $0.9113^{b}$ \\
\hline Height, cm & $155.89 \pm 8.20$ & $|55.7| \pm 8.26$ & $0.8402^{\mathrm{b}}$ \\
\hline $\mathrm{BMI}, \mathrm{kg} / \mathrm{m}^{2}$ & $25.7 \pm 3.9$ & $25.8 \pm 4.4$ & $0.8339^{b}$ \\
\hline Duration of osteoarthritis, years & $4.5 \pm 4.3$ & $4.0 \pm 4.2$ & $0.2637^{\mathrm{b}}$ \\
\hline BPI-Severity average pain & $5.1 \pm 1.0$ & $5.0 \pm 1.0$ & $0.8252^{\mathrm{b}}$ \\
\hline $\begin{array}{l}\text { Use of NSAIDs } \geq 14 \text { days/ } \\
\text { month for } 3 \text { months prior to } \\
\text { study entry }\end{array}$ & $100(56.8 \%)$ & $98(55.4 \%)$ & $0.8304^{c}$ \\
\hline Prior drug therapy & $167(94.6 \%)$ & $158(89.3 \%)$ & $0.0747^{c}$ \\
\hline \multicolumn{4}{|l|}{ Kellgren-Lawrence grade } \\
\hline Grade 0 & $0(0.0 \%)$ & $0(0.0 \%)$ & $0.3944^{d}$ \\
\hline Grade I & $10(5.7 \%)$ & $14(7.9 \%)$ & \\
\hline Grade 2 & $84(47.7 \%)$ & $87(49.2 \%)$ & \\
\hline Grade 3 & $74(42.0 \%)$ & $69(39.0 \%)$ & \\
\hline Grade 4 & $8(4.5 \%)$ & $7(4.0 \%)$ & \\
\hline
\end{tabular}

Notes: Values are presented as the mean \pm SD or $n$ (\%). ${ }^{\text {T}}$ Tested at the significance level of 0.15 . 'Welch's $t$-test. 'Fisher's exact test. 'Wilcoxon rank-sum test.

Abbreviations: BMI, body mass index; BPI, Brief Pain Inventory; NSAIDs, nonsteroidal anti-inflammatory drugs.

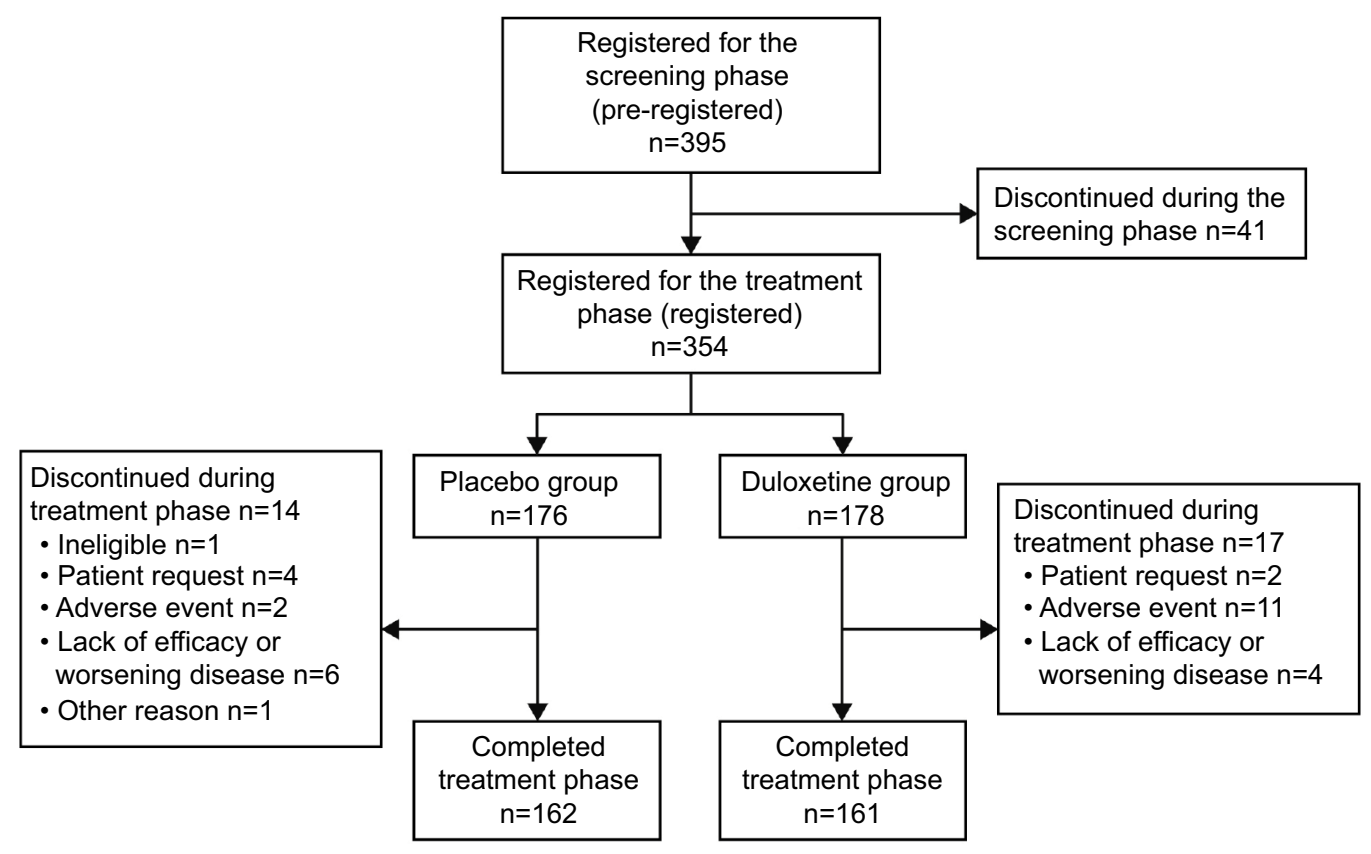

Figure 2 Patient disposition. 
Table 2 Primary and secondary efficacy outcomes

\begin{tabular}{|c|c|c|c|c|}
\hline & $\begin{array}{l}\text { Placebo } \\
(n=176)\end{array}$ & $\begin{array}{l}\text { Duloxetine } \\
(n=\mid 77)\end{array}$ & $\begin{array}{l}\text { Adjusted mean } \\
\text { difference/risk ratio for } \\
\text { duloxetine vs. placebo }\end{array}$ & $P$-value \\
\hline \multicolumn{5}{|l|}{ BPI-Severity } \\
\hline Average pain (primary endpoint) & $-1.80(-2.05$ to -1.56$)$ & $-2.57(-2.81$ to -2.33$)$ & $-0.77(-1.11$ to -0.43$)$ & $<0.000 I^{a}$ \\
\hline $30 \%$ reduction (average pain) & $88(50.0 \%$ [ $42.4 \%$ to $57.6 \%])$ & $124(70.1 \%[62.7 \%$ to $76.7 \%])$ & $1.40(1.17$ to 1.67$)$ & $0.000 \mathrm{I}^{\mathrm{b}}$ \\
\hline $50 \%$ reduction (average pain) & 69 (39.2\% [31.9\% to $46.8 \%])$ & II 4 (64.4\% [56.9\% to $71.4 \%])$ & 1.64 (1.33 to 2.03$)$ & $<0.000 \mathrm{I}^{\mathrm{b}}$ \\
\hline \multicolumn{5}{|l|}{ pain) } \\
\hline Worst pain & $-2.13(-2.42$ to -1.84$)$ & $-2.92(-3.21$ to -2.63$)$ & $-0.79(-1.20$ to -0.38$)$ & $0.0002^{\mathrm{a}}$ \\
\hline Least pain & $-1.05(-1.27$ to -0.83$)$ & $-1.61(-1.83$ to -1.40$)$ & $-0.56(-0.87$ to -0.26$)$ & $0.0003^{\mathrm{a}}$ \\
\hline Pain right now & $-1.52(-1.78$ to -1.26$)$ & $-2.29(-2.55$ to -2.04$)$ & $-0.77(-1.14$ to -0.41$)$ & $<0.000 I^{a}$ \\
\hline \multicolumn{5}{|c|}{ Weekly 24-hour BPI-Severity (patient diaries) } \\
\hline 24-hour average pain score & $-1.79(-2.02$ to -1.57$)$ & $-2.45(-2.68$ to -2.22$)$ & $-0.66(-0.98$ to -0.33$)$ & $<0.000 I^{\mathrm{a}}$ \\
\hline $30 \%$ reduction & 93 (52.8\% [45.2\% to 60.4\%]) & I 28 (72.3\% [65.I\% to $78.8 \%])$ & $1.37(1.16$ to 1.62$)$ & $0.0002^{c}$ \\
\hline $50 \%$ reduction & $68(38.6 \%$ [31.4\% to $46.3 \%])$ & $98(55.4 \%$ [ $47.7 \%$ to $62.8 \%])$ & $1.43(1.14$ to 1.80$)$ & $0.0020^{c}$ \\
\hline Sustained reduction & $88(50.0 \%[42.4 \%$ to $57.6 \%])$ & 126 (7I.2\% [63.9\% to $77.7 \%])$ & $1.42(1.20$ to 1.70$)$ & $<0.000 I^{c}$ \\
\hline 24-hour worst pain score & $-1.97(-2.23$ to -1.70$)$ & $-2.73(-3.00$ to -2.46$)$ & $-0.76(-1.14$ to -0.39$)$ & $<0.000 I^{a}$ \\
\hline \multicolumn{5}{|l|}{ BPI-Interference } \\
\hline General activity & $-1.52(-1.79$ to -1.25$)$ & $-2.42(-2.68$ to -2.15$)$ & $-0.89(-1.27$ to -0.52$)$ & $<\left.0.000\right|^{a}$ \\
\hline Mood & $-1.43(-1.67$ to -1.18$)$ & $-1.95(-2.19$ to -1.70$)$ & $0.52(-0.86$ to -0.17$)$ & $0.0036^{\mathrm{a}}$ \\
\hline Walking ability & $-1.74(-2.01$ to -1.47$)$ & $-2.58(-2.85$ to -2.31$)$ & $-0.84(-1.23$ to -0.46$)$ & $<0.000 I^{a}$ \\
\hline Normal work & $-1.67(-1.94$ to -1.41$)$ & $-2.48(-2.75$ to -2.22$)$ & -0.81 (-1.18 to -0.44$)$ & $<0.000 I^{a}$ \\
\hline Relations with people & $-0.81(-1.03$ to -0.60$)$ & $-1.23(-1.44$ to -1.01$)$ & $-0.4 \mathrm{I}(-0.72$ to $-0.1 \mathrm{I})$ & $0.0070^{\mathrm{a}}$ \\
\hline Sleep & $-1.19(-1.41$ to -0.97$)$ & $-1.65(-1.87$ to -1.43$)$ & $-0.46(-0.78$ to -0.15$)$ & $0.0039^{\mathrm{a}}$ \\
\hline Enjoyment of life & $-1.16(-1.39$ to -0.93$)$ & $-1.78(-2.01$ to -1.55$)$ & $-0.62(-0.94$ to -0.30$)$ & $0.0002^{\mathrm{a}}$ \\
\hline Average of 7 questions & $-1.34(-1.56$ to -1.12$)$ & $-2.01(-2.22$ to -1.79$)$ & $-0.67(-0.97$ to -0.36$)$ & $<0.000 I^{a}$ \\
\hline CGI severity & $-1.22(-1.36$ to -1.08$)$ & $-1.71(-1.85$ to -1.56$)$ & $-0.49(-0.69$ to -0.28$)$ & $<0.000 I^{a}$ \\
\hline PGI-I & 2.84 (2.67 to 3.02$)$ & 2.23 (2.06 to 2.40$)$ & $-0.61(-0.86$ to -0.37$)$ & $<0.000 I^{a}$ \\
\hline EQ-5D & 0.07 (0.05 to 0.09$)$ & $0.12(0.10$ to 0.14$)$ & $0.05(0.02$ to 0.08$)$ & $0.0006^{d}$ \\
\hline \multicolumn{5}{|l|}{ SF-36 } \\
\hline Physical functioning & 6.23 (3.72 to 8.73$)$ & $12.62(10.12$ to 15.12$)$ & 6.39 (2.85 to 9.94$)$ & $0.0004^{d}$ \\
\hline Physical role limitations & $3.66(1.1 I$ to 6.21$)$ & II.44 (8.90, 13.98) & 7.78 (4.18 to II.39) & $<0.000 \mathrm{I}^{\mathrm{d}}$ \\
\hline Bodily pain & $9.63(7.24,12.03)$ & 16.32 (13.93 to 18.72$)$ & 6.69 (3.30 to 10.08$)$ & $0.000 \mathrm{I}^{\mathrm{d}}$ \\
\hline General health perceptions & $1.82(-0.03$ to 3.66$)$ & 5.58 (3.75 to 7.42$)$ & 3.77 (1.16 to 6.37$)$ & $0.0047^{d}$ \\
\hline Vitality & $3.16(1.12$ to 5.20$)$ & 3.99 (1.96 to 6.03$)$ & $0.83(-2.05$ to 3.72$)$ & $0.5698^{d}$ \\
\hline Social functioning & $2.54(0.28$ to 4.80$)$ & $5.66(3.4 \mid$ to 7.92$)$ & $3.12(-0.07$ to 6.31$)$ & $0.0550^{d}$ \\
\hline Emotional role limitations & $0.70(-1.81$ to 3.21$)$ & 6.32 (3.82 to 8.82$)$ & 5.62 (2.06 to 9.17$)$ & $0.0020^{d}$ \\
\hline Mental health & $1.48(-0.47$ to 3.43$)$ & 3.02 ( 1.08 to 4.97$)$ & $1.55(-1.21$ to 4.30$)$ & $0.2697^{d}$ \\
\hline OMERACT-OARSI response rate & $109(61.9 \%$ [54.3\% to $69.1 \%])$ & I 48 (83.6\% [77.3\% to $88.7 \%])$ & 1.35 (1.18 to 1.54$)$ & $<0.000 I^{c}$ \\
\hline
\end{tabular}

Notes: Values are presented as the adjusted mean final change $(95 \% \mathrm{Cl})$ or $\mathrm{n}(\%[95 \% \mathrm{Cl}])$, adjusted mean difference vs. placebo $(95 \% \mathrm{Cl})$, risk ratio vs. placebo $(95 \% \mathrm{Cl})$. aMixed-effect model for repeated measures analysis (adjusted mean difference). ${ }^{b}$ Mantel-Haenszel test (risk ratio). 'Fisher's exact test (risk ratio). ${ }^{\mathrm{A} A n a l y s i s}$ of covariance with last observation carried forward (adjusted mean difference).

Abbreviations: BPI, Brief Pain Inventory; CGI, Clinical Global Impressions; EQ-5D, European Quality of Life Questionnaire-5 Dimension; OMERACT-OARSI, Outcome Measures in Rheumatology Clinical Trials-Osteoarthritis Research Society International; PGI-I, Patient Global Impression of Improvement; SF-36, Medical Outcomes Study 36-Item Short Form Health Survey.

and sustained reductions in average pain; BPI-Severity worst pain, least pain, and pain right now; 24-hour BPI-Severity weekly average pain, including the proportions of patients achieving 30\%, 50\%, and sustained reductions in 24-hour BPI-Severity average pain and 24-hour BPI-Severity worst pain were significantly greater for patients treated with duloxetine compared with placebo (Table 2).
Compared with placebo, there were significantly greater improvements for patients treated with duloxetine in CGI-S and PGI-I at Week 14 (Table 2), adjusted mean changes in EQ-5D, five of the SF-36 subscales (Table 2), BPI-Interference (Table 2), WOMAC total score $(-17.41$ [95\% CI: -19.21 to -15.61 ] vs. -10.45 [95\% CI: -12.25 to -8.65 ]; $P<0.0001$ ), and WOMAC subscale scores (pain, stiffness, 


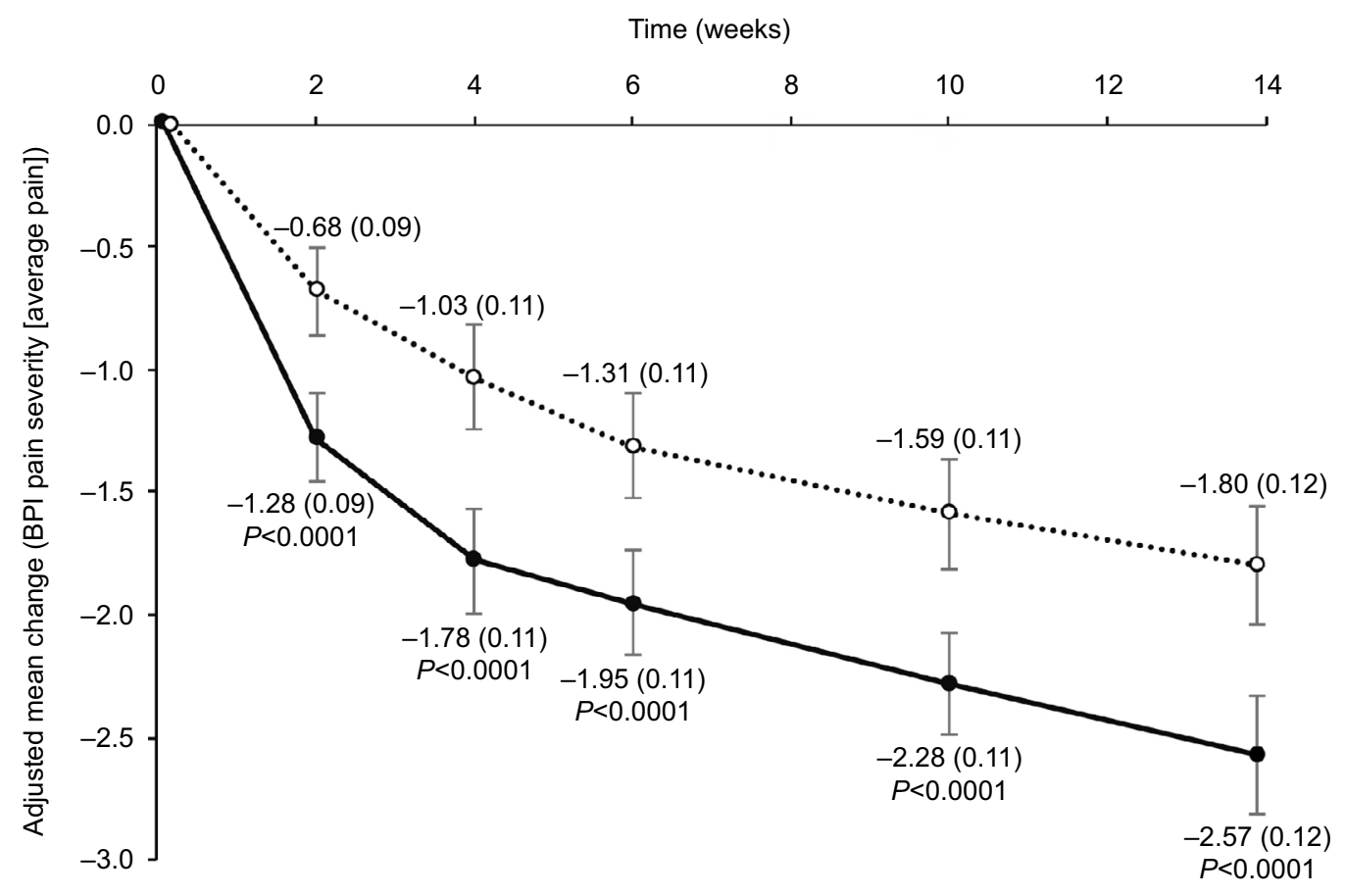

Figure 3 Primary endpoint: change in BPI-Severity average pain.

Notes: Values are shown as the adjusted mean changes from baseline for duloxetine and placebo. Error bars indicate $95 \% \mathrm{Cl}$ and values in brackets indicate standard error. Abbreviation: BPI, Brief Pain Inventory.

and physical function; Figure 4). No significant differences between groups were reported for the SF-36 vitality, social functioning, and mental health subscales.

The OMERACT-OARSI response rate was significantly greater for patients treated with duloxetine compared with placebo (Table 2).

No difference was found in the change in knee ROM between groups. At Week 14, the adjusted mean change from baseline $(95 \% \mathrm{CI})$ in knee ROM was $3.31^{\circ}\left(2.15^{\circ}\right.$ to $\left.4.46^{\circ}\right)$ for duloxetine-treated patients and $3.05^{\circ}\left(1.89^{\circ}\right.$ to $\left.4.21^{\circ}\right)$ for placebo-treated patients $(P=0.7592$, post hoc analysis of covariance, last observation carried forward).

\section{Potential effect of age on BPI-Severity average pain}

The change in BPI-Severity average pain from baseline to Week 14 was examined in a post hoc analysis of patients subdivided by age ( $<65$ and $\geq 65$ years). The adjusted mean change $(95 \% \mathrm{CI})$ was significantly greater for patients treated with duloxetine compared with placebo among patients aged $<65$ years $(-2.34$ [ -2.72 to -1.97$]$ vs. -1.80 [ -2.18 to -1.43$]$; $P=0.0452)$ and among patients aged $\geq 65$ years $(-2.71[-3.03$ to -2.40$]$ vs. $-1.81[-2.12$ to -1.49$] ; P<0.0001)$.

\section{Safety}

The incidences of AEs and ADRs were significantly greater for patients treated with duloxetine than with placebo (Table 3). Compared with placebo, significantly more patients on duloxetine discontinued because of an $\mathrm{AE}$ during the treatment phase (2 [1.1\%] vs. 11 [6.2\%], respectively, $P=0.0202$ ). The most common AE leading to study discontinuation in the duloxetine group was malaise (3/11 patients); all cases were of moderate severity and resolved.

Somnolence, constipation, dry mouth, nausea, malaise, and decreased appetite were more frequent with duloxetine than placebo (Table 3 ). All were mild to moderate in severity (mostly mild). There was one serious $\mathrm{AE}$ in the duloxetine group (cancerous peritonitis) and one in the placebo group (cerebellar tumor). A causal relationship with the study drug was ruled out in both cases. No patients died during the study.

In the duloxetine treatment group, the frequency and type of AEs were similar in patients aged $<65$ years $(65.3 \% ; 47 / 72)$ and $\geq 65$ years $(68.9 \% ; 73 / 106$; Table S1).

No difference was found between groups in the change from baseline in K-L grade. At Week 15, K-L grade had worsened in four (duloxetine) and six (placebo) patients and improved in three (duloxetine) and five (placebo) patients (Table 4). 
A

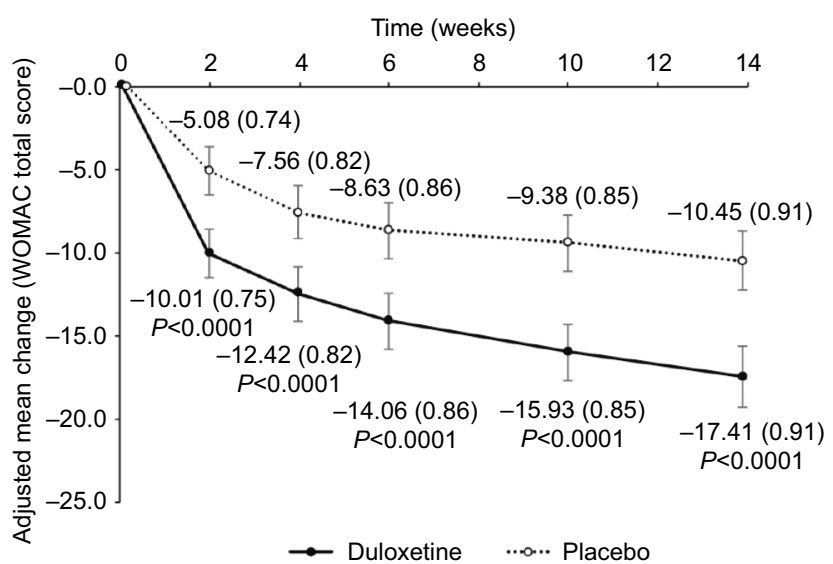

C

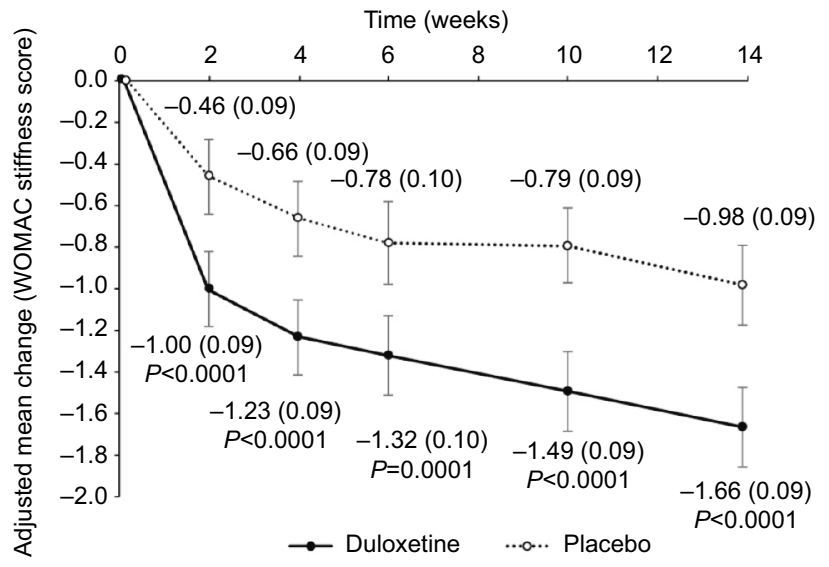

B
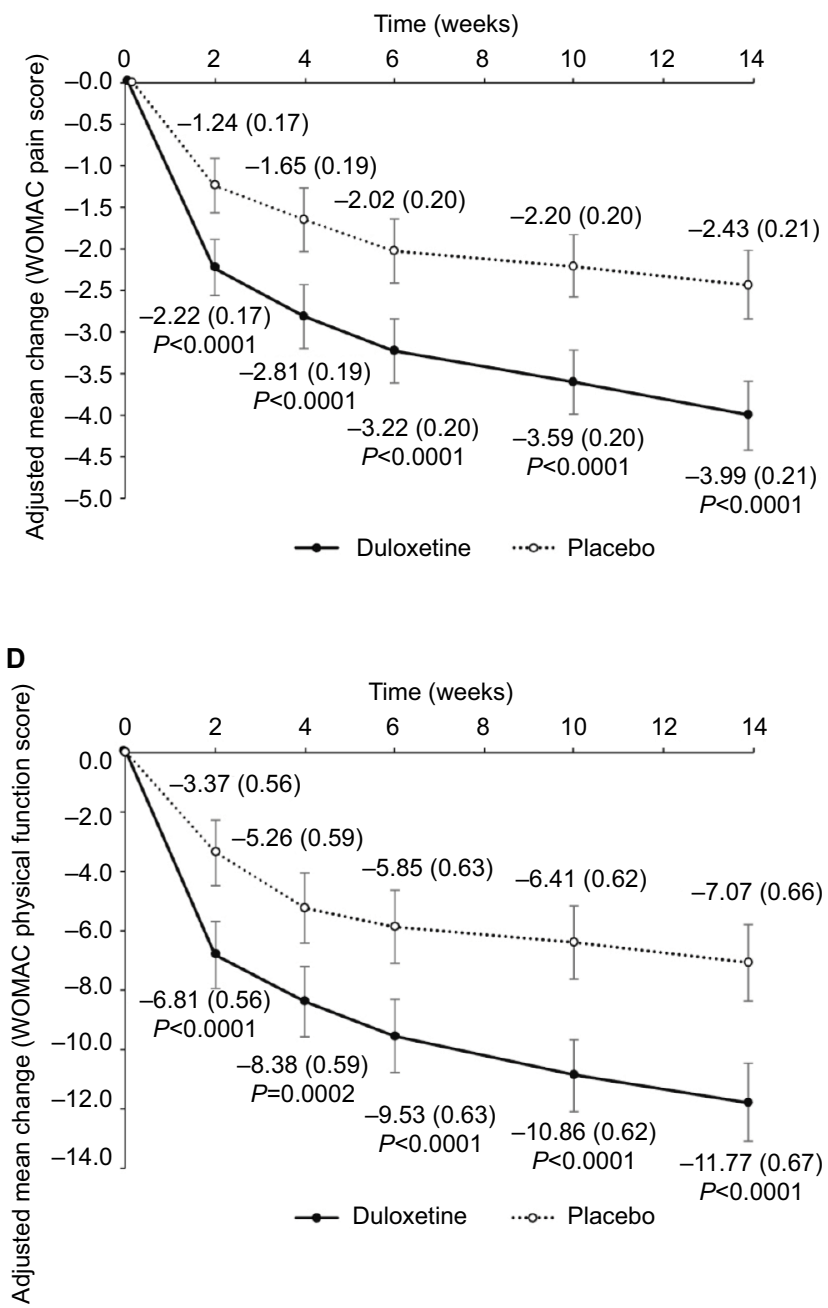

Figure 4 Changes in WOMAC total (A), pain (B), stiffness (C), and physical function (D) scores.

Notes: Values are shown as the adjusted mean changes from baseline for duloxetine and placebo. Error bars indicate $95 \% \mathrm{Cl}$ and values in brackets indicate standard error. Abbreviation: WOMAC, Western Ontario and McMaster Universities osteoarthritis index.

Table 3 Adverse events and adverse drug reactions

\begin{tabular}{|c|c|c|c|c|}
\hline & $\begin{array}{l}\text { Total } \\
(n=354)\end{array}$ & $\begin{array}{l}\text { Placebo } \\
(n=176)\end{array}$ & $\begin{array}{l}\text { Duloxetine } \\
(n=178)\end{array}$ & $P$-value ${ }^{a}$ \\
\hline All AEs & & 98 (55.7\%) & $120(67.4 \%)$ & 0.0287 \\
\hline All ADRs & & $26(14.8 \%)$ & 77 (43.3\%) & $<0.0001$ \\
\hline \multicolumn{5}{|c|}{ AEs in $\geq 5 \%$ of patients in either group } \\
\hline Nasopharyngitis & $55(15.5 \%)$ & $28(15.9 \%)$ & $27(15.2 \%)$ & 0.8841 \\
\hline Somnolence & $30(8.5 \%)$ & $6(3.4 \%)$ & $24(13.5 \%)$ & 0.0009 \\
\hline Constipation & $22(6.2 \%)$ & $3(1.7 \%)$ & $19(10.7 \%)$ & 0.0006 \\
\hline Dry mouth & $22(6.2 \%)$ & $3(1.7 \%)$ & $19(10.7 \%)$ & 0.0006 \\
\hline Nausea & $19(5.4 \%)$ & I $(0.6 \%)$ & $18(10.1 \%)$ & $<0.000$ I \\
\hline Malaise & $14(4.0 \%)$ & $2(1.1 \%)$ & $12(6.7 \%)$ & 0.0113 \\
\hline Contusion & $16(4.5 \%)$ & $7(4.0 \%)$ & $9(5.1 \%)$ & 0.7991 \\
\hline Decreased appetite & $10(2.8 \%)$ & I (0.6\%) & $9(5.1 \%)$ & 0.0198 \\
\hline
\end{tabular}

Notes: ${ }^{a}$ Fisher's exact test.

Abbreviations: $A D R$, adverse drug reaction; $A E$, adverse event. 
Table 4 Kellgren-Lawrence grade at baseline and at Week I5

\begin{tabular}{llllll}
\hline & Week I5 & \multicolumn{3}{l}{ Baseline } & \\
\cline { 3 - 6 } & & Grade I & Grade 2 & Grade 3 & Grade 4 \\
\hline Placebo & Grade 0 & 0 & 0 & 0 & 0 \\
(n=176) & Grade I & 9 & 0 & 1 & 0 \\
& Grade 2 & I & 79 & 4 & 0 \\
& Grade 3 & 0 & 4 & 67 & 0 \\
& Grade 4 & 0 & 0 & 1 & 8 \\
Duloxetine & No observation & 0 & 1 & 1 & 0 \\
(n=178) & Grade 0 & 0 & 0 & 0 & 0 \\
& Grade I & 12 & 2 & 0 & 0 \\
& Grade 2 & 2 & 79 & 1 & 0 \\
& Grade 3 & 0 & 2 & 67 & 0 \\
& Grade 4 & 0 & 0 & 0 & 7 \\
& No observation & 0 & 5 & 1 & 0
\end{tabular}

Note: Shading indicates no change in Kellgren-Lawrence grade.

All other safety assessments were consistent with the known safety profile of duloxetine (data not shown).

\section{Discussion}

Findings from this double-blind randomized controlled trial comparing duloxetine and placebo for treatment of pain associated with knee OA demonstrated superior efficacy for duloxetine in the reduction in BPI-Severity average pain at Week 14. Compared with placebo, patients treated with duloxetine had significant improvements in all measures of pain, stiffness, and physical function (BPI-Severity, 24-hour BPI-Severity, WOMAC scores, and OMERACT-OARSI response rate). The reduction in pain with duloxetine was associated with improvements in most HRQoL total and component scores, including HRQoL specific to OA (WOMAC). Importantly, improvements in pain and physical function with duloxetine were not associated with any X-ray abnormalities (K-L grade), which might indicate overuse after pain reduction. In addition, the AEs observed were consistent with the known safety profile for duloxetine and no new safety concerns were reported. Together, these data suggest that duloxetine is an effective treatment for pain in Japanese patients with knee OA.

Overall, our findings in this Japanese population are not discrepant with, and extend, findings from placebo-controlled clinical trials of duloxetine conducted in US/Europe and China, although our patients were older than in those studies, reflecting Japan's aging society. ${ }^{9-11,24}$ Improvements in pain severity in our 14-week study of up to $60 \mathrm{mg}$ /day duloxetine were similar to those in US/Europe, which were shorter (10-13 weeks) and included higher duloxetine doses (up to $120 \mathrm{mg} /$ day). ${ }^{9,10}$ Improvements in pain severity in our study were also similar to a 13-week study of knee and hip
OA in China, with an upper limit of $60 \mathrm{mg}$ /day duloxetine. ${ }^{11}$ To further explore the effect of duloxetine on pain severity in patients with knee OA, we compared effect sizes for duloxetine from this study with previous studies. ${ }^{9,10}$ Effect size was calculated as the adjusted mean difference in the change in BPI-Severity average pain from baseline between duloxetine and placebo divided by the estimated SD in each study, where the estimated SD was based on the number of patients in each group at the last visit and the standard error of the adjusted mean in each study. The effect size in our study $(0.51)$ was similar to those from previous studies with duloxetine $(0.55,0.43) .{ }^{9}, 10$

Similar to previous studies, ${ }^{9-11}$ treatment with duloxetine was associated with significant improvements in physicianand patient-reported physical functioning and HRQoL. These results suggest that alleviation of pain with duloxetine had positive effects on patients' physical functioning, and subsequently on patients' mental, emotional, and social QoL. However, no significant differences were found between the duloxetine and placebo groups for vitality, social functioning, and mental health SF-36 subscales. The relevance of this finding is unclear. In contrast to our study, the US/Europe studies found no differences compared with placebo in the SF-36 subscales of general health perceptions and emotional role limitations, and several BPI-Interference subscales (mood, relations with people). ${ }^{9,10}$ In addition, one study ${ }^{10}$ found significant improvement in the SF-36 mental health subscale. Compared with the study conducted in China, ${ }^{11}$ there were significant improvements in a larger number of HRQoL items in our study. For example, significant improvements in the BPI-Interference items "relations with people," "sleep," and "enjoyment of life" were observed in our study, but not in the study from China. The reasons for observed differences in HRQoL among the various studies are likely multifactorial and related to, but not limited to, differences in patient demographic and clinical characteristics, ethnic and cultural differences, and treatment duration and dose.

Overall, the frequency and severity of AEs in duloxetinetreated patients in this study were consistent with the known safety profile of duloxetine and the AEs observed in clinical trials of duloxetine for knee $\mathrm{OA}$ in other populations. ${ }^{9-11}$ Among the most frequent AEs, somnolence, constipation, dry mouth, nausea, malaise, and decreased appetite were more frequent in the duloxetine group. Moreover, no apparent differences were found in the frequency or type of AEs between patients aged $<65$ and $\geq 65$ years. This finding is particularly relevant given the aging population in Japan and that pain relief is more likely to be required in patients 
with knee OA as they age. ${ }^{3}$ However, the incidences of AEs and ADRs were significantly greater and significantly more patients discontinued treatment because of an $\mathrm{AE}$ in the duloxetine group than in the placebo group.

Pain in patients with knee OA results partly from the disease process and partly from pain avoidance strategies, such as reduced physical activity and maladaptive movement, which further exacerbate pain and contribute to impaired HRQoL..$^{7,25,26}$ Duloxetine may provide an opportunity to interrupt this cycle by reducing pain and allowing patients to increase their physical activity. Moreover, if pain is reduced to a level that enables patients to receive various physical therapies, further improvements in motor function, and subsequently in HRQoL, may be expected. ${ }^{25}$ Alternatively, alleviation of pain in response to treatment may exacerbate joint deterioration by allowing patients to overuse their joints. To our knowledge, this is the first study to assess whether pharmacological pain therapy may contribute to X-ray abnormalities or alterations in knee joint mobility. K-L grading was used to examine whether the reduction in pain worsened knee OA, potentially as an effect of increased physical activity. ${ }^{27}$ However, we did not observe any marked changes in K-L grade in patients treated with duloxetine or placebo. Further, there were no reports of patients whose condition worsened with overactivity after receiving duloxetine. Despite this, it is important to note that cartilage thinning cannot be detected using plain X-ray images and a 14-week treatment phase may not have been sufficient time to observe marked changes in $\mathrm{X}$-ray features.

The strengths of this study are that it was a double-blind randomized trial that included multiple measures of efficacy. Although randomized trials are designed to minimize bias and establish efficacy, the treatment schedule, patient visits, and restrictions on concomitant therapy and comorbidities (e.g., patients with psychiatric disorders) may not reflect real-world clinical practice. In addition, the relatively short-treatment duration (14 weeks) did not enable long-term assessment of the efficacy or safety of duloxetine. Although the study was conducted in a single population, the efficacy and safety findings were consistent with studies conducted in USA/Europe and China, suggesting that the findings are generalizable to other study populations.

\section{Conclusion}

In conclusion, duloxetine showed superior clinical efficacy to placebo in pain reduction in Japanese patients with knee OA. This study also revealed greater improvements in HRQoL in duloxetine-treated patients compared with placebo. The safety of duloxetine was consistent with its known safety profile. No marked changes were found in joint structure on plain X-ray images in duloxetine-treated patients within the relatively short study duration. Together, these findings suggest that duloxetine may be considered for the alleviation of pain in Japanese patients with pain due to knee OA.

\section{Acknowledgments}

The authors thank all clinical investigators and patients for their participation in the study. The authors thank Marion Barnett and Nicholas Smith of Edanz Medical Writing for providing medical writing support, which was funded by Shionogi \& Co., Ltd. Editing of the final manuscript was provided by Serina Stretton of ProScribe-Envision Pharma Group and funded by Shionogi \& Co., Ltd.

This work was supported by Shionogi \& Co., Ltd., Eli Lilly Japan K.K., and Eli Lilly and Company. Shionogi \& Co., Ltd., Eli Lilly Japan K.K., and Eli Lilly and Company contributed to study design, data analysis, and writing of the manuscript.

\section{Author contributions}

All authors were study investigators and contributed toward data analysis and interpretation, drafting and critically revising the manuscript, and approving the final version for publication, and agree to be accountable for all aspects of the work.

\section{Disclosure}

YU has received honoraria/consulting fees, travel support, fees for participation in review activities, fees for writing assistance, medicine, equipment, and administrative support, and/or grants from Shionogi \& Co., Ltd.; Astellas Pharma Inc.; Asahi Kasei Pharma Corporation; Chugai Pharmaceutical Co., Ltd.; Daiichi Sankyo Co., Ltd.; Kaken Pharmaceutical Co., Ltd.; Nippon Zoki Pharmaceutical Co., Ltd.; Seikagaku Corporation; Taisho Toyama Pharmaceutical Co., Ltd.; Teijin Pharma Limited, Pfizer Japan Inc.; Japan Tissue Engineering Co., Ltd.; Ayumi Pharmaceutical Corporation; Hisamitsu Pharmaceutical Co., Inc.; HOYA Technosurgical Corporation; Mochida Pharmaceutical Co., Ltd.; Eli Lilly Japan K.K.; Olympus Terumo Biomaterials Corporation; Janssen Pharmaceutical K.K; Ministry of Economy, Trade and Industry; Japan Science and Technology Agency; Japan Society for the Promotion of Science; Japan Sports Medicine Foundation; and the Terumo Foundation for Life Sciences and Arts. HE and LA are employees of and hold stock in Eli Lilly 
Japan K.K. YK is an employee of Shionogi \& Co., Ltd. HI, TT, and TO are employees of and hold stock in Shionogi \& Co., Ltd. SK has received grants, honoraria, reviewing fees, payment for lectures, fees for writing assistance, medicines, equipment, and/or administrative support from Shionogi \& Co., Ltd.; MSD K.K.; Asahi Kasei Pharma Corporation; Eli Lilly Japan K.K.; Eisai Co., Ltd.; Ono Pharmaceutical Co., Ltd.; Kaken Pharmaceutical Co., Ltd.; Kowa Pharmaceutical Co., Ltd.; Showa Yakuhin Kako Co., Ltd.; Johnson \& Johnson K.K.; Daiichi Sankyo Co., Ltd.; Taisho Toyama Pharmaceutical Co., Ltd.; Takeda Pharmaceutical Co., Ltd.; Terumo Corporation; Nippon Zoki Pharmaceutical Co., Ltd.; Hisamitsu Pharmaceutical Co., Inc.; Pfizer Japan Inc.; Janssen Pharmaceutical K.K.; Ayumi Pharmaceutical Corporation; Taiho Pharmaceutical Co., Ltd.; Chugai Pharmaceutical Co., Ltd.; Teijin Pharma Limited; Astellas Pharma Inc.; Nippon Shinyaku Co., Ltd.; Stryker Japan K.K.; Tsumura \& Co.; and Otsuka Pharmaceutical Co., Ltd.

\section{References}

1. GBD 2015 Disease and Injury Incidence and Prevalence Collaborators. Global, regional, and national incidence, prevalence, and years lived with disability for 310 diseases and injuries, 1990-2015: a systematic analysis for the Global Burden of Disease Study 2015. Lancet. 2016;388(10053):1545-1602.

2. Litwic A, Edwards MH, Dennison EM, Cooper C. Epidemiology and burden of osteoarthritis. Br Med Bull. 2013;105(1):185-199.

3. Muraki S, Oka H, Akune T, et al. Prevalence of radiographic knee osteoarthritis and its association with knee pain in the elderly of Japanese population-based cohorts: the ROAD study. Osteoarthritis Cartilage. 2009;17(9):1137-1143.

4. Jinks C, Jordan K, Croft P. Osteoarthritis as a public health problem: the impact of developing knee pain on physical function in adults living in the community: (KNEST 3). Rheumatol. 2007;46(5):877-881.

5. Sharma L, Cahue S, Song J, Hayes K, Pai YC, Dunlop D. Physical functioning over three years in knee osteoarthritis: role of psychosocial, local mechanical, and neuromuscular factors. Arthritis Rheum. 2003;48(12):3359-3370.

6. Muraki S, Akune T, Oka H, et al. Association of radiographic and symptomatic knee osteoarthritis with health-related quality of life in a population-based cohort study in Japan: the ROAD study. Osteoarthritis Cartilage. 2010;18(9):1227-1234.

7. Bindawas SM, Vennu V, Al Snih S. Differences in health-related quality of life among subjects with frequent bilateral or unilateral knee pain: data from the Osteoarthritis Initiative study. J Orthop Sports Phys Ther. 2015;45(2):128-136.

8. McAlindon TE, Bannuru RR, Sullivan MC, et al. OARSI guidelines for the non-surgical management of knee osteoarthritis. Osteoarthritis Cartilage. 2014;22(3):363-388.

9. Chappell AS, Desaiah D, Liu-Seifert H, et al. A double-blind, randomized, placebo-controlled study of the efficacy and safety of duloxetine for the treatment of chronic pain due to osteoarthritis of the knee. Pain Pract. 2011;11(1):33-41.
10. Chappell AS, Ossanna MJ, Liu-Seifert H, et al. Duloxetine, a centrally acting analgesic, in the treatment of patients with osteoarthritis knee pain: a 13-week, randomized, placebo-controlled trial. Pain. 2009;146(3):253-260.

11. Wang G, Bi L, Li X, et al. Efficacy and safety of duloxetine in Chinese patients with chronic pain due to osteoarthritis: a randomized, double-blind, placebo-controlled study. Osteoarthritis Cartilage. 2017;25(6):832-838.

12. Perahia DG, Kajdasz DK, Desaiah D, Haddad PM. Symptoms following abrupt discontinuation of duloxetine treatment in patients with major depressive disorder. J Affect Disord. 2005;89(1-3):207-212.

13. Altman R, Asch E, Bloch D, et al. Development of criteria for the classification and reporting of osteoarthritis. Classification of osteoarthritis of the knee. Diagnostic and Therapeutic Criteria Committee of the American Rheumatism Association. Arthritis Rheum. 1986;29(8): 1039-1049.

14. Sheehan D, Lecrubier Y. M.I.N.I.: International Neuropsychiatric Interview. Japanese 5.0.0 ed. Tokyo: Seiwa Shoten Co., Ltd; 2003.

15. Posner K, Brown GK, Stanley B, et al. The Columbia-Suicide Severity Rating Scale: initial validity and internal consistency findings from three multisite studies with adolescents and adults. Am J Psychiatry. 2011;168(12):1266-1277.

16. Uki J, Mendoza T, Cleeland CS, Nakamura Y, Takeda F. A brief cancer pain assessment tool in Japanese: the utility of the Japanese Brief Pain Inventory-BPI-J. J Pain Symptom Manage. 1998;16(6):364-373.

17. Tan G, Jensen MP, Thornby JI, Shanti BF. Validation of the Brief Pain Inventory for chronic nonmalignant pain. J Pain. 2004;5(2):133-137.

18. Konno S, Oda N, Ochiai T, Alev L. Randomized, double-blind, placebocontrolled phase III trial of duloxetine monotherapy in Japanese patients with chronic low back pain. Spine. 2016;41(22):1709-1717.

19. Guy W. ECDEU Assessment Manual for Psychopharmacology. Rockville, MD: National Institute of Mental Health (U.S.). Psychopharmacology Research Branch. Division of Extramural Research Programs; 1976.

20. Brooks R. EuroQol: the current state of play. Health Policy. 1996;37(1):53-72.

21. Fukuhara S, Bito S, Green J, Hsiao A, Kurokawa K. Translation, adaptation, and validation of the SF-36 Health Survey for use in Japan. J Clin Epidemiol. 1998;51(11):1037-1044.

22. Hashimoto H, Hanyu T, Sledge CB, Lingard EA. Validation of a Japanese patient-derived outcome scale for assessing total knee arthroplasty: comparison with Western Ontario and McMaster Universities osteoarthritis index (WOMAC). J Orthop Sci. 2003;8(3):288-293.

23. Pham T, Van Der Heijde D, Lassere M, et al. Outcome variables for osteoarthritis clinical trials: the OMERACT-OARSI set of responder criteria. J Rheumatol. 2003;30(7):1648-1654.

24. Wang ZY, Shi SY, Li SJ, et al. Efficacy and safety of duloxetine on osteoarthritis knee pain: a meta-analysis of randomized controlled trials. Pain Med. 2015;16(7):1373-1385.

25. Kuru Çolak T, Kavlak B, Aydoğdu O, et al. The effects of therapeutic exercises on pain, muscle strength, functional capacity, balance and hemodynamic parameters in knee osteoarthritis patients: a randomized controlled study of supervised versus home exercises. Rheumatol Int. 2017;37(3):399-407.

26. Ruhdorfer A, Wirth W, Eckstein F. Association of knee pain with a reduction in thigh muscle strength-a cross-sectional analysis including 4553 osteoarthritis initiative participants. Osteoarthritis Cartilage. 2017;25(5):658-666.

27. Emrani PS, Katz JN, Kessler CL, et al. Joint space narrowing and Kellgren-Lawrence progression in knee osteoarthritis: an analytic literature synthesis. Osteoarthritis Cartilage. 2008;16(8):873-882. 


\section{Supplementary materials}

\section{Allowed and prohibited interventions/ treatments during the study}

Concomitant use of analgesics, muscle relaxants, antidepressants, sedatives, and benzodiazepines was prohibited afterVisit 1. However, zopiclone, eszopiclone and zolpidem started before Visit 1 were permitted for continued use after Visit 1, provided no changes were made in their dosing regimens. Drugs with analgesic effects (except for tramadol hydrochloride) were permitted between Visits 2 and 8, but only as rescue medication for the underlying disease or as treatment for adverse events for up to 3 consecutive days and up to a cumulative total of 20 days. However, the use of these drugs was prohibited from the day before the efficacy evaluation day to the end of the evaluation on that day. The upper limit of the daily dose was the approved dose. Aspirin could be used after Visit 1, at a maximum daily dose of $325 \mathrm{mg}$, to prevent thrombus or embolism formation. Interventions, such as intra-articular hyaluronic acid or steroid injections, arthrocentesis and exercise therapy, were prohibited after Visit 1. Non-pharmacological medical therapy aimed at pain relief or sedation for the underlying disease and started $\geq 3$ months before Visit 1 was permitted, provided there were no changes in the therapy. However, non-orthotic treatment was prohibited from the day before the efficacy evaluation day until the end of the evaluations.

\section{6-Item Short Form Health Survey (SF-36) and European Quality of Life Questionnaire-5 Dimension (EQ-5D) quality of life scales}

In the SF-36, patients evaluated their health status by answering 36 questions measuring the following eight subscales

\section{Box SI List of Institutional Review Boards}

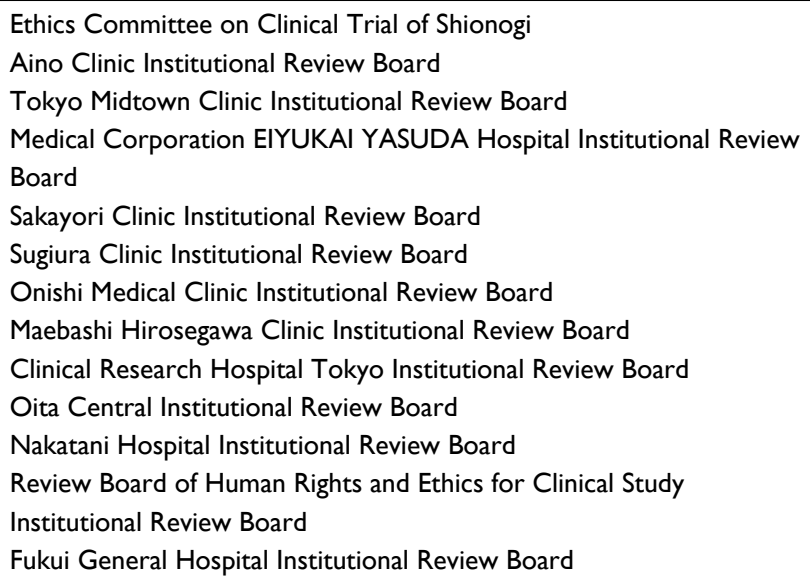

by rating on a $0-100$ scale: physical functioning, physical role functioning, emotional role functioning, general health perceptions, social role functioning, bodily pain, vitality, and mental health. In the EQ-5D, patients estimated their health status in five domains (mobility, self-care, usual activities, pain/discomfort, and anxiety/depression) on a 3-point rating scale.

\section{X-ray imaging}

Plain X-ray images of the knee joints were taken at Visit 1 and 8 (i.e, after completing the tapering phase) or at discontinuation during the tapering phase. X-ray images were graded using the Kellgren-Lawrence classification. ${ }^{1}$

\section{Assessment of knee joint range of motion (ROM)}

Knee ROM was evaluated at Visit 1 and 7 (ie, after completion of the treatment phase) or at discontinuation during the treatment phase. The change in the ROM (degrees) after treatment was compared between the two groups.

\section{Sample size calculation}

The target sample size for this study was determined based on the results of overseas Phase III clinical studies in patients with osteoarthritis of the knee. ${ }^{2,3}$ In a meta-analysis of the results from both studies, the difference in the change in Brief Pain Inventory (BPI)-Severity average pain between the duloxetine and placebo groups was estimated to be -0.9 , with a standard deviation of 2.0. Because a dose increase from 60 to $120 \mathrm{mg}$ was performed in the duloxetine group, this estimated size effect may have included the dose-increase effect. Therefore, by estimating a $20 \%$ smaller effect size in the present study and by ensuring a power of $\geq 90 \%$ for the between-group comparisons using two-sample $t$-tests at a two-sided significance level of $5 \%$, the target sample size was determined to be 340 patients, with 170 patients per group.

\section{Secondary analysis of the primary end point}

Secondary analysis of the primary endpoint was performed using analysis of covariance with the change from baseline as a response variable, treatment group as a fixed effect, and the baseline value as a covariate. Missing data were imputed using the last observation carried forward approach (LOCF), baseline observation carried forward approach (BOCF), or the modified BOCF. In the modified BOCF approach, the BOCF was used for patients who discontinued the study 
Table SI Incidence of adverse events (in $\geq 2 \%$ of patients in any group) according to treatment received and age group

\begin{tabular}{|c|c|c|c|c|}
\hline & \multicolumn{2}{|c|}{ Patients $<65$ years } & \multicolumn{2}{|c|}{ Patients $\geq 65$ years } \\
\hline & Placebo & Duloxetine & Placebo & Duloxetine \\
\hline & $(n=68)$ & $(n=72)$ & $(n=108)$ & $(n=106)$ \\
\hline Overall & 39 (57.4\%) & 47 (65.3\%) & 59 (54.6\%) & $73(68.9 \%)$ \\
\hline Nasopharyngitis & 16 & 12 & 12 & 15 \\
\hline Somnolence & 2 & 12 & 4 & 12 \\
\hline Nausea & I & 9 & 0 & 9 \\
\hline Constipation & I & 8 & 2 & 11 \\
\hline Malaise & I & 6 & I & 6 \\
\hline Decreased appetite & I & 6 & 0 & 3 \\
\hline Headache & 4 & I & 1 & 0 \\
\hline Dry mouth & 0 & 4 & 3 & 15 \\
\hline Back pain & 3 & 1 & 5 & 0 \\
\hline Diarrhea & I & 3 & 0 & 3 \\
\hline Contusion & 2 & I & 5 & 8 \\
\hline Abdominal discomfort & 2 & I & 2 & 1 \\
\hline ALT increase & 0 & 0 & I & 8 \\
\hline AST increase & 0 & 0 & 1 & 7 \\
\hline
\end{tabular}

Notes: Values are presented as $\mathrm{n}(\%)$ or $\mathrm{n}$.

Abbreviations: ALT, alanine aminotransferase; AST, aspartate aminotransferase.

because of adverse events or lack of efficacy and the LOCF was used for patients who discontinued the study for other reasons. Differences between duloxetine and placebo are presented as the adjusted mean difference between the two groups with $95 \% \mathrm{CI}$.

\section{Analyses of secondary efficacy and safety endpoints}

The $30 \%$ pain reduction, $50 \%$ pain reduction, and sustained pain reduction rates were compared between the duloxetine and placebo group, by calculating the number of responders and the response rate for each treatment group, and by using the Mantel-Haenszel test adjusted for the allocation factors. The $30 \%$ pain reduction and $50 \%$ pain reduction were defined as the proportion of patients in whom the BPI-Severity average pain at the last observation was reduced by $30 \%$ and $50 \%$, respectively, from baseline. Sustained pain reduction was defined as the proportion of patients showing a reduction in BPI-Severity average pain of $\geq 30 \%$ from baseline to the last observation and at a time point before the last observation, with a sustained reduction of $\geq 20 \%$ from baseline at all of the intervening time points.

Mixed-effects model repeated measures analysis was used to compare the changes from baseline to Week 14 in BPISeverity, the extent of interference with functioning using the BPI (BPI-Interference), 24 hours average pain severity score (weekly average score), 24 hours worst pain severity score (weekly average score), Clinical Global Impressions severity, Patient's Global Impression of improvement, and
Western Ontario and McMaster Universities Osteoarthritis Index subscales (pain, stiffness, difficulty in performing daily activities) and total scores. The $30 \%$ pain reduction, $50 \%$ pain reduction, sustained pain reduction in 24 hours average pain severity score (weekly average score), and the Outcome Measures in Rheumatology Clinical Trials-Osteoarthritis Research Society International response rates were compared between the duloxetine and placebo groups using a Fisher's exact test. The changes in SF-36 and EQ-5D from baseline to Week 14 of treatment were compared between the duloxetine and placebo groups using analysis of covariance. Differences between duloxetine and placebo are presented as the adjusted mean difference between the two groups with $95 \%$ CI. For BPI/Weekly 24 hours average pain score $30 \%$ reduction, $50 \%$ reduction, sustained reduction, and Outcome Measures in Rheumatology Clinical Trials-Osteoarthritis Research Society International (OMERACT-OARSI) criteria response rates, the $95 \%$ CIs in each group were determined using the Clopper-Pearson method. A Fisher's exact test was used to compare the rates of adverse events and adverse drug reactions between the two groups.

\section{References}

1. Kellgren JH, Lawrence JS. Radiological assessment of osteo-arthrosis. Ann Rheum Dis 1957;16(4):494-502.

2. Chappell AS, Ossanna MJ, Liu-Seifert H, et al. Duloxetine, a centrally acting analgesic, in the treatment of patients with osteoarthritis knee pain: a 13-week, randomized, placebo-controlled trial. Pain 2009;146(3):253-260.

3. Chappell AS, Desaiah D, Liu-Seifert H, et al. A double-blind, randomized, placebo-controlled study of the efficacy and safety of duloxetine for the treatment of chronic pain due to osteoarthritis of the knee. Pain Pract 2011;11(1):33-41. 
The Journal of Pain Research is an international, peer reviewed, open access, online journal that welcomes laboratory and clinical findings in the fields of pain research and the prevention and management of pain. Original research, reviews, symposium reports, hypothesis formation and commentaries are all considered for publication
The manuscript management system is completely online and includes a very quick and fair peer-review system, which is all easy to use. Visit http://www.dovepress.com/testimonials.php to read real quotes from published authors. 